\section{Ultrastructure of acrokeratoelastoidosis}

\section{Editor}

Acrokeratoelastoidosis (AKE) is a genodermatosis characterized by small, firm papules or plaques on the sides of the hands and feet, ${ }^{1}$ and it was first described in 1953 by Costa, in a Brazilian patient.

It is a rare disease, and both autosomal dominant and sporadic forms have been observed. ${ }^{2}$ It is characterized by multiple hyperkeratotic papules on the palms, soles and dorsum of the hands and feet. Typical histopathological findings in AKE include hyperkeratosis, acanthosis with fragmented and decreased elastic fibres in reticular dermis (elastorrhexis). ${ }^{3}$

Electron microscopy (EM) findings in AKE are limited.

A 45-year-old white woman presented with small asymptomatic palmar papules that emerged at the beginning of adolescence. These papules were waxy and in great number along the borders of the hands, but were also present in the flexures of the fingers (Fig. 1a). In detail, the dermatoglyphics were not affected (Fig. 1b), suggesting a dermal involvement. The patient had a bureaucratic job and her activities and hobbies did not require constant use of hands. The feet were not affected. She denied a positive family history.

Light microscopy with HE staining showed unspecific findings with compact hyperkeratosis and marked acanthosis.
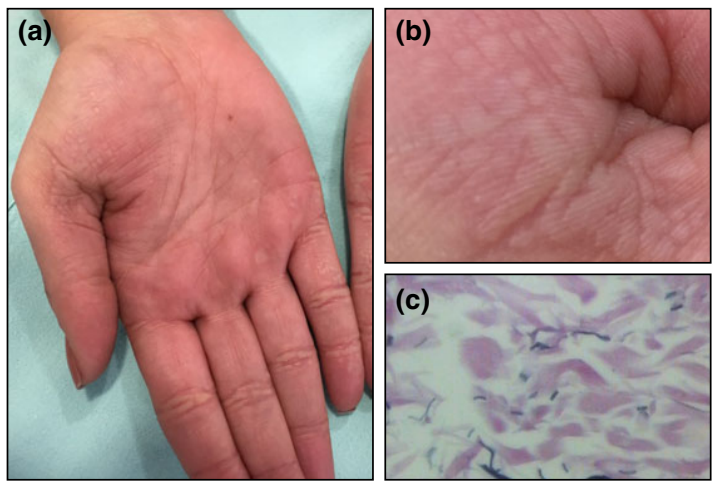

Figure 1 (a) waxy papules in the palmar surface. (b) detail showing persistence of dermatoglyphics. (c) light microscopy with Weigert staining showing diminished and fragmented elastic fibres $(\times 400)$.
Weigert stain revealed reduced dermal elastic fibres (Fig. 1c) which were thinner and fragmented.

Transmission EM showed easily decreased thickness of the elastic fibres (Fig. 2a,b), with light surface indentations (Fig. 2b arrows). Marked fragmentation of elastic fibres could also be observed (Fig. 2c) as well as areas of granular degeneration. The fibre core displayed gross and irregular black spots. Collagen bundles showed normal aspect.

Scanning EM demonstrated the fragmented aspect of the elastic fibres (Fig. 2d), which resembled rooster crests. Higher magnifications revealed the same indentations observed by TEM (Fig. 2e).

AKE is a disease that belongs to the group of genetic marginal keratoderma and affects primarily the lateral palmoplantar regions.

It is a rare autosomal dominant disease, with also sporadic cases ${ }^{2}$ such this case. It is frequently seen in infancy or adolescence, but has also been reported in adults. ${ }^{2-5}$ The pathogenesis of the disease is still unknown, and there is no predominance of gender or race. ${ }^{1}$ The clinical presentation is characterized by multiple yellowish keratotic papules, sometimes translucent, measuring 2-4 $\mathrm{mm}$ in diameter, sometimes umbilicated, located symmetrically on the lateral margin of the hands and feet, only palmar involvement was already described, similarly to our case. The edge of the fingers, the wrists and the anteroposterior surfaces of the legs may also be affected. Some authors have reported an increase in the number of papules over time. Furthermore, there may be an association with hyperhidrosis. ${ }^{2,6}$

The most common histopathological findings are hyperkeratosis, mild acanthosis and alterations in the elastic fibres of the dermis, which are diminished and fragmented (elastorrhexis). ${ }^{3}$ Previous TEM findings suggest a defect in the production and fragmentation of elastic fibres in the dermis. ${ }^{7}$

TEM showed decreased thickness of elastic fibres, with discreet surface indentations. Marked fragmentation of elastic fibres could also be observed, similar to light microscopy. The fibre core displayed gross and irregular black spots. These findings corroborate the results reported in previous studies. ${ }^{3-6}$ Collagen bundles seemed normal.

SEM showed the fragmented aspect of the elastic fibres, resembling a rooster crest, which was not previously described in literature. Higher magnifications reveal the same indentations observed by TEM.

These findings are different from other diseases with malformations of elastic fibres, such as pseudoxanthoma elasticum, with most fibres exhibiting rounded aggregates, ${ }^{8}$ but may be 

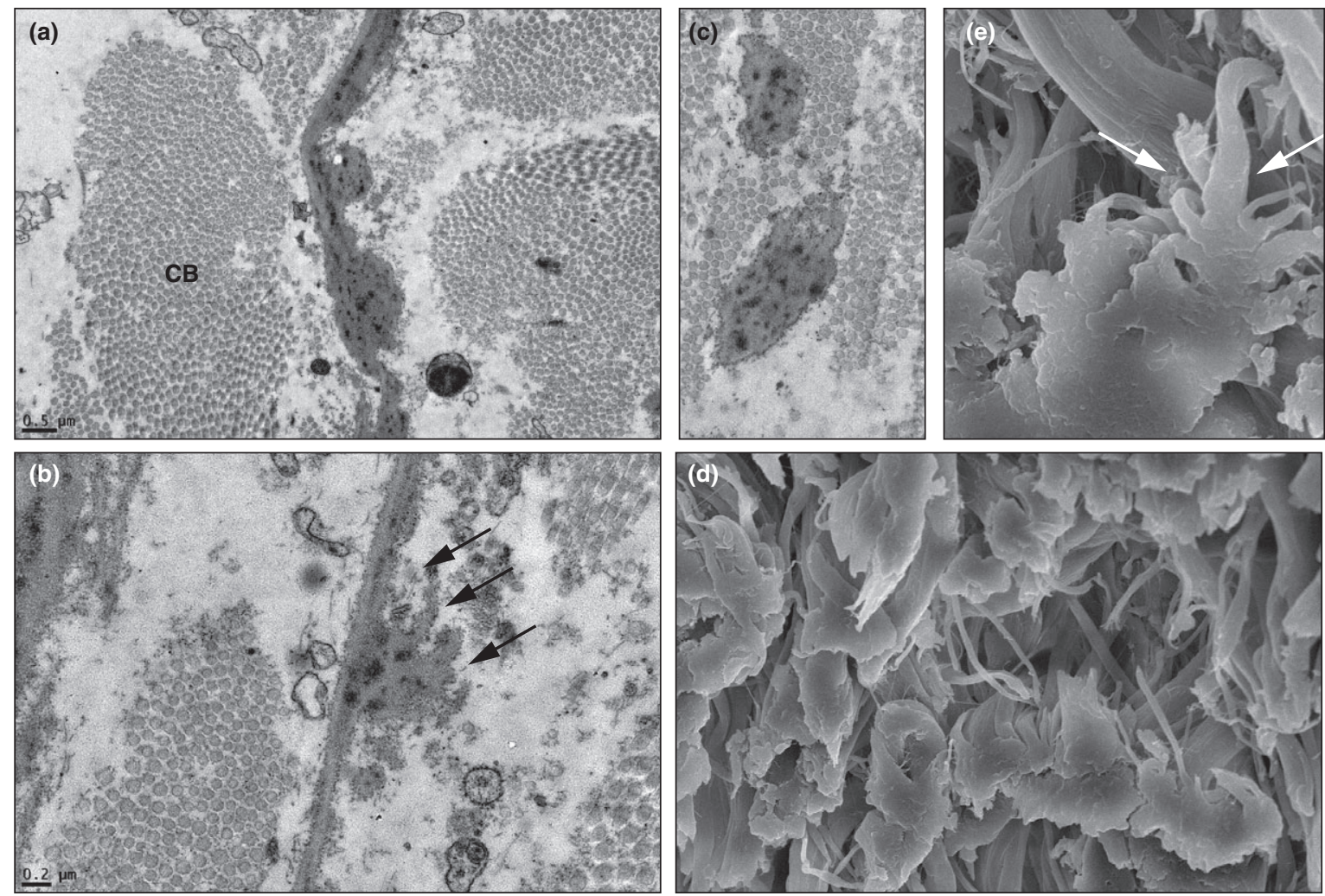

Figure 2 Transmission electron microscopy (a) thin elastic fibre with a normal collagen bundle (CB) $(\times 20$ 000). (b) thin elastic fibre with surface indentations (arrows) $(\times 40$ 000). (c) fragmented elastic fibres ( $\times 30$ 000). Scanning electron microscopy (d) fragmented elastic fibres $(\times 1800)$. (e) elastic fibre with indentations (arrows) resembling rooster crest $(\times 3700)$.

similar to other elastorrhexic conditions, such as primary anetodermia, which shows granular degeneration of elastic fibres. ${ }^{9}$

\section{J.F. Lopes, ${ }^{1}$ H.L. de Almeida Jr., ${ }^{1,2, \star}$ R.R. da Cunha Filho, ${ }^{3}$ B. Viganó Lattman, ${ }^{3}$ L.A. Suíta de Castro ${ }^{4}$}

${ }^{1}$ Catholic University of Pelotas, Pelotas, Brazil, ${ }^{2}$ Federal University of Pelotas, Pelotas, Brazil, ${ }^{3}$ Western University of Santa Catarina, Joaçaba, Brazil, ${ }^{4}$ EMBRAPA-CPA-CT, Pelotas, Brazil

${ }^{*}$ Correspondence: H.L. de Almeida Jr. E-mail: hiramalmeidaj@@hotmail.

com

\section{References}

1 Lewis KG, Bercovitch L, Dill SW, Robinson-Bostom L. Acquired disorders of elastic tissue: Part I. Increased elastic tissue and solar elastotic syndromes. J Am Acad Dermatol 2004; 51: 1-21.

2 Costa MC, Demarch EB, Hertz A, Pereira FBC, Azulay DR. Caso para diagnóstico. An Bras Dermatol 2011; 86: 1222-1223.

3 Bogle MA, Hwang LY, Tschen JA. Acrokeratoelastoidosis. J Am Acad Dermatol 2002; 47: 448-451.
4 Meziane M, Senouci K, Ouidane Y et al. Acrokeratoelastoidosis. Dermatol Online J 2008; 14: 11

5 van Steensel MA, Frank J. Focal acral hyperkeratosis and acrokeratoelastoidosis: birds of a feather? J Eur Acad Dermatol Venereol 2009; 23: 1113

1114.

6 van Steensel MA, Verstraeten VL, Frank J. Acrokeratoelastoidosis with nail dystrophy: a coincidence or a new entity? Arch Dermatol 2006; 142: 939941.

7 Fiallo P, Pesce C, Brusasco A, Nunzi E. Acrokeratoelastoidosis of Costa: a primary disease of the elastic tissue? J Cutan Pathol 1998; 25: 580-582.

8 De Almeida HL Jr, de Almeida MG, Jorge VM, de Abreu LB. Ultrastructural aspects of pseudoxanthoma elasticum. An Bras Dermatol 2017; 92: 527-530.

9 Göebel-Pinto JB, de Almeida HL, de Castro LAS, Rocha NM. Ultrastructural aspects of primary anetoderma. J Cutan Pathol 2017; 44: 786-789.

DOI: $10.1111 / j d v .14660$ 